Lessons learnt Our programme addresses the imbalance of Paediatric experience in GP training when compared to the Paediatric proportion of a typical GP's workload.

Message for others We feel the programme would benefit to GP trainees across the country and could easily be introduced in other deaneries.

\section{G520(P) WITHDRAWN}

\section{G521(P) BABY FRIENDLY PREVENTION AND MANAGEMENT OF NEONATAL HYPOGLYCAEMIA ON POSTNATAL WARDS}

${ }^{1} \mathrm{C}$ Matthews, ${ }^{2} \mathrm{E}$ Sage, ${ }^{3} \mathrm{P}$ Reynolds. 'Paediatric Surgery, Evelina Children's Hospital, London, UK; ${ }^{2}$ Paediatrics, The Royal Marsden Hospital, London, UK; ${ }^{3}$ Neonatal Intensive Care Unit, St Peter's Hospital, London, UK

10.1136/archdischild-2015-308599.472

Context The project was undertaken at a level 3 NICU in a DGH in Surrey, UK, involving junior and senior doctors, midwives, nursery nurses, pharmacists and the pathology department.

Problem Staff anecdotally felt the hypoglycaemia operational threshold was too high, monitors were inaccurate, blood sugar level (BSL) tests too frequent and management inconsistent. Treatment with formula feed supplements was hindering efforts to establish breastfeeding and prolonging stays.

Assessment of problem and analysis of its causes Initial assessment involved an audit of current practice against Baby-Friendly Initiative standards and interviews with 62 multidisciplinary team members to explore concerns and collate ideas for improvement. To check equipment accuracy, 50 samples were tested for blood glucose on both the portable metres and blood gas machine, which found on average glucosmeters under-read by $0.5 \mathrm{mmol} / \mathrm{L}$ (range $0-2.0 \mathrm{mmol} / \mathrm{L}$ ). A review of literature and other UK trust's policies was carried out to identify best practice and alternatives to formula-feed supplementation. It was recognised that new equipment would need to be used for BSL monitoring and all staff would need to be trained in any policy changes.

Intervention New guidelines lowered the working definition of hypoglycaemia, limited the number of BSL tests performed by stopping routine measurement of post-feed BSLs, and changed first line treatment of moderate hypoglycaemia (BSL $>2 \mathrm{mmol} / \mathrm{L}$ ) from formula top-up to dextrose gel. A new proforma with stepby-step management plan and built in escalation was designed with the midwives. The pathology department is acquiring more accurate glucosmeters for the trust; in the meantime a "glucose only" micro-sample setting on NICU gas machine has been set up.

Study design Audit of practice pre- and post- new management guidelines was undertaken to measure the number of needle pricks each baby had, number of days each infant at risk of hypoglycaemia was monitored for, supplementation rates, the number of babies treated for hypoglycaemia who were discharged breast feeding and still doing so at 3 months, and the number of infants admitted to NICU for management of hypoglycaemia.

Strategy for change Management of hypoglycaemia guidelines were re-written and a new proforma produced within 6 months. This was sent to all Neonatal Consultants and senior midwives for review. All midwives and nursery nurses were trained by the authors and the point-of-care team in the new guidelines, how to take a capillary blood gas and how to run a glucose only sample. Audit of practice as outlined above was carried out for infants treated 6 months before and 6 months after guideline implementation, and the results fed back to the Neonatal Consultant body.

Measurement of improvement Audit of practice showed postimplementation the average number of BSL tests per baby reduced by $73 \%$ (15 to 4 pricks) and average monitoring time reduced by $52 \%$ - saving $520 \mathrm{hrs} /$ month staff time. Breastfeeding rates at 3 months post discharge have doubled and provisional data shows a $38 \%$ reduction in SCU/NICU admissions for neonatal hypoglycaemia.

Effects of changes Changes implemented meant more accurate blood sugar analysis, fewer needle pricks for infants, shorter admission time for infants at risk of hypoglycaemia, and decreased use of formula supplementation which has enabled the trust to apply for Level 3 Baby Friendly accreditation. Some senior staff were concerned the new proforma was too complicated; this was addressed through face-to-face consultation.

Lessons learnt The most important lesson was the necessity of having all members of the multi-disciplinary team involved in the project from the beginning.

Message for others Change to the status quo to improve patient care and experience is possible by quantifying a long-standing anecdotal problem and implementing evidence based practice.

\section{G522(P) IMPROVING PAEDIATRIC MALARIA CARE IN A LOW RESOURCE SETTING}

1,2S Bihari, 1,2L Waldegrave, 'P Fillie, 'R Samuels, 1,2P Gibson. 'Paediatrics, Ola During Children's Hospital, Freetown, Sierra Leone; ${ }^{2}$ Global Links Programme, Royal College of Paediatrics and Child Health, London, UK

\subsection{6/archdischild-2015-308599.473}

Context The improvement took place in a large paediatric referral hospital in West Africa.

Problem Malaria is one of the leading causes of morbidity and mortality for children attending the hospital. There was no uniform guidance or set standard on the management of malaria. As a result, treatment varied from doctor to doctor, patient to patient.

Assessment of problem and analysis of its causes 40 clinical notes per week were randomly selected for 5 weeks (Weeks 1-5). The data collected compared current practice against the standard expected for the management of a child presenting with a fever/malaria. Multiple areas where the standards were not met were identified at all stages of the child's journey from presenting to the hospital with fever all the way to discharge home or death.

Intervention A new malaria guideline and training package was developed. The guideline included a flow chart, investigations and $1^{\text {st }}$ and $2^{\text {nd }}$ line treatment options, a drug treatment table and guidance on how to prepare and prescribe the treatment on a drug chart. These guidelines were approved by the National Malaria Control Programme and then implemented across the hospital, through teaching and training workshops.

Study design The study used two cohort groups of patients; The first cohort included patients who were admitted to the hospital prior to the intervention the second cohort included patients who were admitted to the hospital after the intervention. The data analysed compared the findings between both groups. 
Strategy for change The results were disseminated to the doctors and then again to the malaria quality care committee. Ninety nine percent $(99 \%)$ of clinical staff received training on the new guidelines; new laboratory forms were introduced to aid the rapid diagnosis of malaria at triage and in the emergency room and every ward had malaria job aid posters. The training of all staff took approximately 5 weeks to complete.

Measurement of improvement Using the same data collection methods, 40 clinical notes per week were selected, from a total of 5 weeks (Weeks 6-10); these weeks represented the start of the training/implementation; half way through training; end of training of all staff and 2 further follow on weeks after all training had been completed.

Over a period of 6 months: The proportion of patients with fever tested for malaria increased from $76 \%$ to $94 \%$. The proportion of malaria cases correctly classified according to the admitting doctor increased from 52\% to 94\%. The proportion of severe malaria cases given appropriate first line treatment increased from $50 \%$ to $95 \%$. Overall mortality attributed to Malaria decreased from $38 \%$ to $25 \%$

Effects of changes The improvement resulted in a higher quality of care being delivered to patients with malaria, in particular, better access to diagnostic tests, more accurate classification of malaria, improved documentation of malaria medication on prescription charts and improved sign off of doses given.

Lessons learnt The data collection process used (i.e. retrospective collection of a random selection on notes) may have given us biassed results and affected the quality of the outcome data. It was also difficult to measure certain specific areas of improvement. Prospective assessment and data collection would be a better way to measure the effects of change.

Message for others By engaging professionals, senior and junior, who are all involved in the care of a child and placing the child at the centre of the process and maintaining that focus on the child is an important way to improve the quality of their care, even in low resource settings.

\section{G523(P) THE PAEDIATRIC RETURN TO WORK PROGRAMME}

A Copeman, A Belfitt. Paediatrics, New Cross Hospital, Wolverhampton, UK

\subsection{6/archdischild-2015-308599.474}

Context We developed a half day programme of fully immersive simulation in a purpose built high fidelity centre to address confidence and training needs of paediatric ST doctors returning to clinical work after a break in practice.

Problem Returning to work after a break from clinical practice is daunting. Many trainees have up to a year away from clinical work on maternity leave or out of programme experience (OOP). Trainees undertaking research have up to 3 years away. Feeling under-confident, stressed and de-skilled can result in underperformance which ultimately puts patient safety at risk.

Assessment of problem and analysis of its causes Research suggests that the length of time away from clinical practice is a key factor affecting a doctor's performance when returning to practice - the longer the time out of clinical medicine in years the poorer their performance. $67 \%$ of doctors absent from practice for more than 18 months require moderate to considerable reeducation or training.

The RCPCH has written guidence to support trainees returning to work however simulation as a tool facilitating this process does not feature. We conducted a literature review but were unable to find any stand alone UK simulation programmes specifically aimed at helping paediatricians return to clinical practice safely.

Intervention A Paediatric Return To Work Simulation Programme was developed and piloted to address trainees confidence, skills and knowledge.

We used scenarios mapped to the RCPCH curriculum covering key areas of emergency management, safeguarding, leadership, communication skills and human factors.

Study design The effectiveness of our programme was assessed using pre and post programme questionnaires (Table 1). Two questionnaires were undertaken immediately before and after simulation training and a final questionnaire was undertaken 3 months later following their return to clinical practice.

Our primary outcome was aimed at evaluating the trainees confidence levels managing neonatal, paediatric and safeguarding problems and whether these improved following our simulation programme. We were also interested in the overall learning experience perceived by the trainees.

Strategy for change All OOP trainees within the deanery were emailed information about the pilot programme. 25 trainees participated in the pilot from September 2013-2014.

Measurement of improvement $88 \%$ of trainees expressed concern about returning to clinical practice prior to the simulation. Reasons given included lack of knowledge, technical skills, confidence, competence and senior responsibility.

Likert scales were used to assess confidence in managing acute paediatric, neonatal, and safeguarding problems pre and post intervention.

Results -confidence All trainees found the programme beneficial $100 \%$ found the training useful preparation for returning to work.

$100 \%$ agreed the experience learned from the training had been useful in their clinical work.

Free text responses were all overwhelmingly positive.

Effects of changes This programme improves confidence and the perceived ability of trainees to manage acute clinical problems. This positive effect appears to be sustained.

As a result of this pilot "The Paediatric Return to Work Simulation Programme" has secured funding and been approved regionally for trainees to attend prior to their return to clinical work.

Lessons learnt Organisations must have clear support and training in place to enable trainees to return to clinical work safely. Paediatric simulation can effectively facilitate this process. Future development would be aimed at identifying whether these positive effects result in an improvement in clinical practice and safety.

\begin{tabular}{|c|c|c|c|}
\hline & Pre Simulation & Post Simulation & 3 month follow up \\
\hline $\begin{array}{l}\text { Confidence managing } \\
\text { acute paediatrics }\end{array}$ & $28.00 \%$ & $96.00 \%$ & $100.00 \%$ \\
\hline $\begin{array}{l}\text { Confidence managing } \\
\text { neonates }\end{array}$ & $32.00 \%$ & $76.00 \%$ & $100.00 \%$ \\
\hline $\begin{array}{l}\text { Confidence managing } \\
\text { safeguarding }\end{array}$ & $44.00 \%$ & $92.00 \%$ & $75.00 \%$ \\
\hline
\end{tabular}

